Journal of Computer Science 4 (11): 897-902, 2008

ISSN 1549-3636

(C) 2008 Science Publications

\title{
A Heapify Based Parallel Sorting Algorithm
}

\author{
${ }^{1}$ Mwaffaq A. Abu Al hija, ${ }^{2}$ Arwa Zabian, ${ }^{3}$ Sami Qawasmeh and ${ }^{4}$ Omer H. Abu Al haija \\ ${ }^{1}$ Department of Computer Science, Hail University, Hail, Saudi Arabia \\ ${ }^{2}$ Department of Computer Science, Jadara University, Irbid, Jordan \\ ${ }^{3}$ Department of Computer Science, Irbid National University, Irbid, Jordan \\ ${ }^{4}$ Department of Computer Science, Irbid, Jordan
}

\begin{abstract}
Quick sort is a sorting algorithm whose worst case running time is $\theta\left(\mathrm{n}^{2}\right)$ on an input array of $n$ numbers. It is the best practical for sorting because it has the advantage of sorting in place. Problem statement: Behavior of quick sort is complex, we proposed in-place $2 \mathrm{~m}$ threads parallel heap sort algorithm which had advantage in sorting in place and had better performance than classical sequential quick sort in running time. Approach: The algorithm consisted of several stages, in first stage; it splits input data into two partitions, next stages it did the same partitioning for prior stage which had been spitted until $2 \mathrm{~m}$ partitions was reached equal to the number of available processors, finally it used heap sort to sort respectively ordered of non internally sorted partitions in parallel. Results: Results showed the speed of algorithm about double speed of classical Quick sort for a large input size. The number of comparisons needed was reduced significantly. Conclusion: In this study we had been proposed a sorting algorithm that uses less number of comparisons with respect to original quick sort that in turn requires less running time to sort the same input data.
\end{abstract}

Key words: Parallel sorting, heap sort, Quick sort, in-place sorting

\section{INTRODUCTION}

Sorting is one of the most well studied problems in computer science because it is fundamental in many applications. Quick sort ${ }^{[1]}$ is typically the fastest sorting algorithm, basically due to better cache behavior among other factors. However, the worst case running time for Quick sort is $\mathrm{O}\left(\mathrm{n}^{2}\right)$ for an input size $\mathrm{n}$, which is unacceptable for large data sets. In this study, we propose PSA Partition and Swap Algorithm, which reduces the time needed for sorting with respect to Quiksort in the worst case. A comparison between Quick sort and PSA algorithm in terms of running time is performed; the simulation results show that PSA is faster than Quick sort in the worst case for sorting the same input data size. Our algorithm is based on partitioning the input data to different partitions then using Heapify procedure in parallel to sort each partition. The heap is a very fundamental data structure used in many application programs. A heap is a data structure that stores an array in a binary tree maintaining two properties: the first is that the value stored in every node is smaller than or equal to the value of its children. The second property is that the binary tree must be complete. This implies that the complete binary tree with height $\mathrm{h}$ has between $2^{\mathrm{h}}$ and $2^{\mathrm{h}+1}-1$ node. So, the height of a binary tree of $\mathrm{n}$ nodes is $\mathrm{O}\left(\log _{2} \mathrm{n}\right)$. The basic operations of the heap are BuildHeap and Heapify. Heapify operation arranges the heap to restore its heap property.

\section{MATERIALS AND METHODS}

Partition and Swap Algorithm (PSA): Given an array of size n, PSA divides the array into four sub-arrays in two steps to facilitate the sorting and to reduce the number of comparisons needed. The algorithm works in three phases the first partition phase, the second partition phase and finally the Heapify phase.

In the first partition phase the algorithm divides the array $A^{[n]}$ into two sub-arrays $A_{1}, A_{2}$ based on the middle value midval, in a manner that all the elements of $A_{1}$ (or denoted left) are smaller than the midval and all the elements of $\mathrm{A}_{2}$ (or denoted as right) are greater than the (midval), if the data must be ordered in an ascendant manner. (If the data must be ordered in descendent manner, all the elements of $A_{1}$ must be greater than the midval and all the elements of $\mathrm{A}_{2}$ must be smaller). In the second partition phase, the partitioning in the first partition phase is repeated for each of $A_{1}, A_{2}$. Where $A_{1}$ is divided into two subarrays 
$A_{11}, A_{1 r}$ and $A_{2}$ is divided into two sub arrays $A_{2 l}, A_{2 r}$ then the elements of these four sub arrays are ordered as in the previous phase. The third phase, is heavily phase in which the resultant subarrays $\mathrm{A}_{11}, \mathrm{~A}_{1 \mathrm{r}}, \mathrm{A}_{2 \mathrm{l}}, \mathrm{A}_{2 \mathrm{r}}$ are organized using heavily procedure into sorted subarrays.

\section{First partition phase:}

- Given an array A of size $\mathrm{n}$ find the middle location of the $n$ elements the pivot can be find by $\lceil n / 2\rceil$. Step 1 in the flowchart

- For each part (left and right to the pivot) find the max and min values (step 2 in the flowchart)

- Find the mid value midval that can be calculated as following:

$$
\left\lfloor L_{\text {max }}+L_{\text {min }}+R_{\text {max }}+R_{\text {min }} / 4\right\rfloor
$$

- For each element in the two subarrays $A_{1}$ (left), $A_{2}$ (right) do the following comparisons (if the array is ordered in an ascendant manner) For $i, j$ are two pointers,
$\mathrm{A}^{[0]}, \mathrm{A}^{[\mathrm{n}]}$ denote the first and last element in the array

\section{Swap procedure:}

for $\mathrm{i}=0$ to $n / 2, j=n$ to $n / 2 \mathrm{do}$ :

if $\mathrm{A}^{[0]}=\mathrm{L}_{\max }>$ midval and

$$
\begin{gathered}
R_{\min }=A^{[n]}<L_{\max } \\
\text { Swap }\left(R_{\min }, L_{\max }\right) \\
\text { if } \mathrm{i} \neq \mathrm{j} \\
\mathrm{i}=\mathrm{i}+1, \mathrm{j}=\mathrm{j}+1 \\
\text { Return if }
\end{gathered}
$$

Else End

Else

$\mathrm{i}=\mathrm{i}+1$ and Return if

The procedure stops when $\mathrm{i}=\mathrm{j}$, that means the two pointers are encountered and all the elements on the left and on the right have been visited and compared. This condition is important to ensure the correctness of the algorithm and to ensure that all the elements have been compared. In the case of $\mathrm{n}$ being an odd number, we take $\lfloor n / 2\rfloor$ to find the mid location (pivot location),

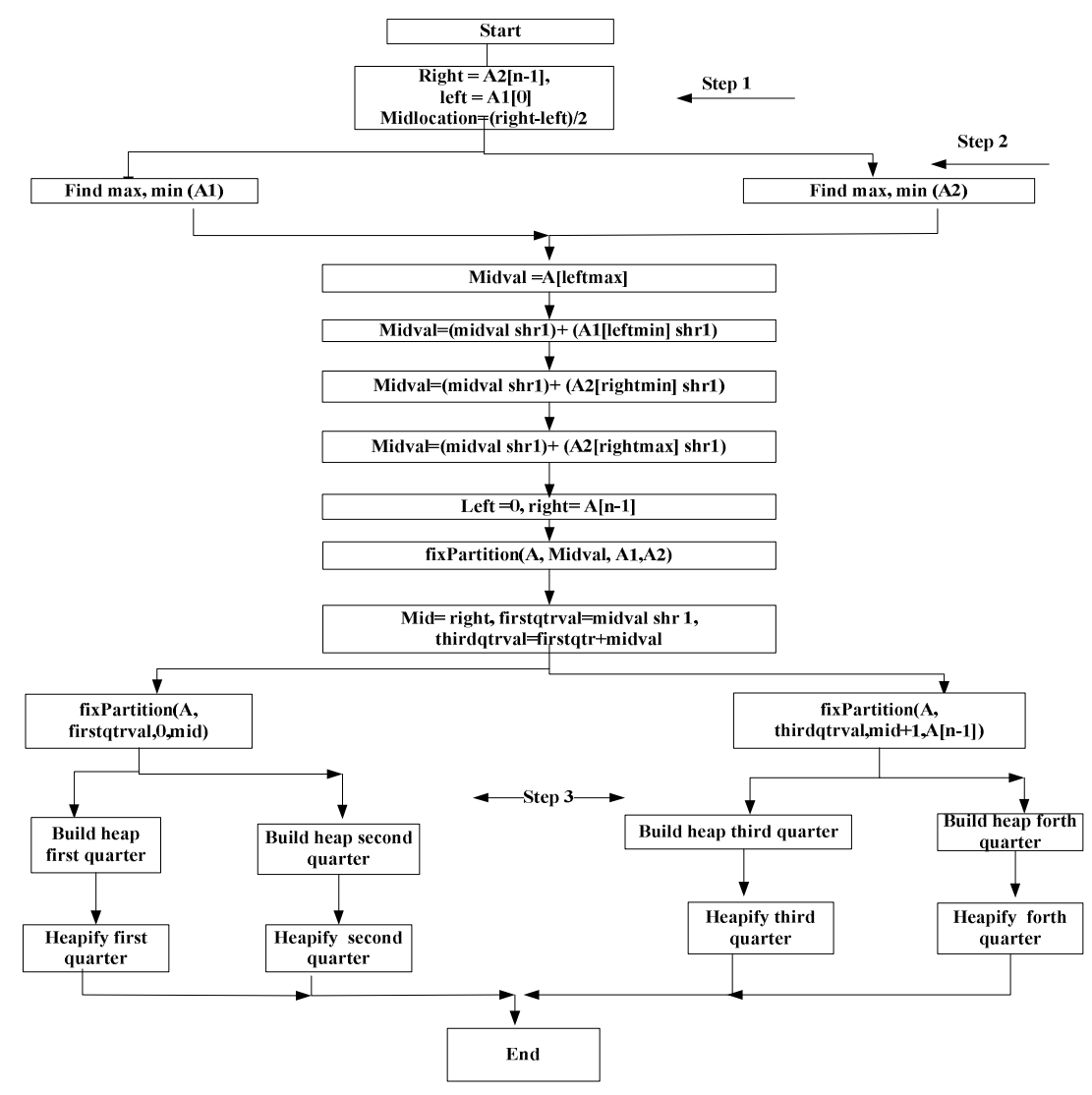

Fig. 1: The flow chart of PSA algorithm 


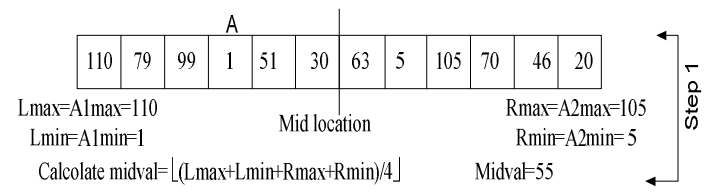

\begin{tabular}{|l|l|l|l|l|l|l|l|l|l|l|l|}
\hline 20 & 79 & 99 & 1 & 51 & 30 & 63 & 5 & 105 & 70 & 46 & 110 \\
\hline
\end{tabular}

Swap values $>$ midval to the right \& swap values < = midval to the left using 1 thread

\begin{tabular}{|l|l|l|l|l|l|l|l|l|l|l|l|}
\hline 20 & 46 & 99 & 1 & 51 & 30 & 63 & 5 & 105 & 70 & 79 & 110 \\
\hline
\end{tabular}

\begin{tabular}{|l|l|l|l|l|l|l|l|l|l|l|l|}
\hline 20 & 46 & 5 & 1 & 51 & 30 & 63 & 99 & 105 & 70 & 79 & 110 \\
\hline
\end{tabular}

\begin{tabular}{rl|l|l|l|l|l|}
\hline 20 & 46 & 5 & 1 & 51 & 30 \\
\hline
\end{tabular}

\begin{tabular}{|l|l|l|l|l|l|}
\hline 63 & 99 & 105 & 70 & 79 & 110 \\
\hline \multicolumn{3}{|c|}{ Right=A2 }
\end{tabular}

Fig. 2: PSA algorithm/first partition phase

in which case the right will have more elements than the left and the pointer i must continue to visit the elements until it encounters $j$.

Second partition phase: The algorithm starts working in parallel in dividing the two subarrays $A_{1}, A_{2}$ to four consecutive partitions $\mathrm{A}_{11}, \mathrm{~A}_{1 \mathrm{r}}, \mathrm{A}_{2 \mathrm{l}}, \mathrm{A}_{2 \mathrm{r}}$ and the swap procedure will be repeated for the following counters in $A_{1}$ for $i=0$ to $n / 4, j=n / 2$ to $n / 4$ and for $A_{2}$ the counter will be: for $i=n / 2$ to $3 n / 2, j=n$ to $j=3 n / 2$.

At the end of this phase we obtain four subarrays in such a manner that:

All the elements of $A_{11}<$ all the elements of $A_{1 r}$ and all the elements of $A_{21}<$ all the elements of $A_{2 r}$

Heapify phase: In this phase, we use heavily procedure on all the four resultant sub-arrays to organize them in sorted subarrays.

Figure 1 shows the flowchart that describes how PSA work. In the first step of the flowchart the initial array is divided into two sub-arrays and the midvallocation is found. In the second step, the min and max values for each partition are found and a series of swap operations are done. Step 3 indicates the last phase that is heavily phase.

Example: Figure 2 shows a numerical example of how PSA works in an array of size $n=12$. In Fig. 2, A is divided into two sub-arrays $\mathrm{A}_{1}, \mathrm{~A}_{2}$ where the bold items

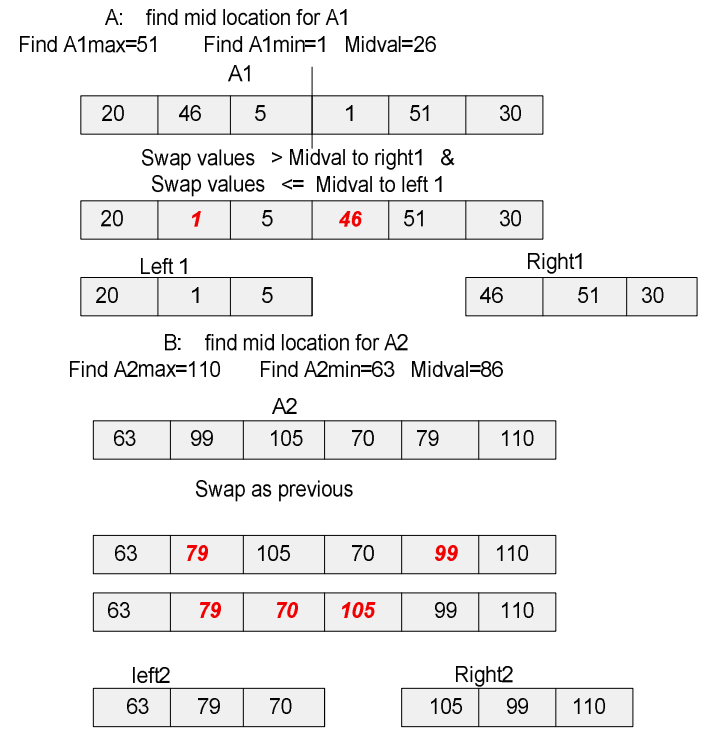

Fig. 3: PSA algorithm/merge in place

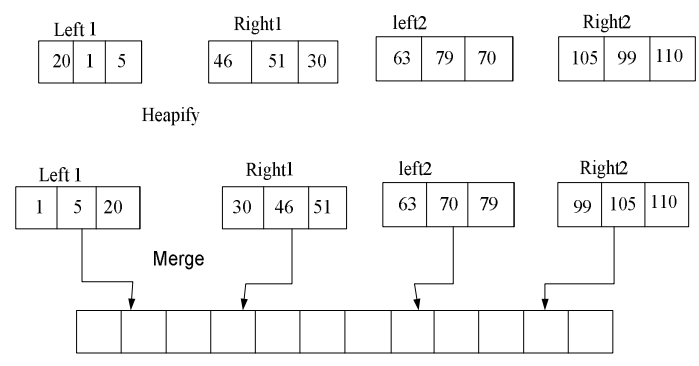

Fig. 4: PSA Algorithm/ Heapify Phase

represent the swapped elements. In part A of Fig. 3, the sub-array $A_{1}$ is divided, to left1 and right 1 . In part $B$, $\mathrm{A}_{2}$ is divided into two sub-arrays left 2 , right 2 . In Fig. 4, it is shown how the four sorted sub-arrays are inserted in their location into the final sorted array.

\section{RESULTS}

To study the effectiveness of our algorithm PSA with respect to Quick sort and other sorting algorithms, we have used turbo Delphi to implement both Quick sort and PSA. Where we wrote a generic code that could run on both programs. Turbo Delphi is an integrated development environment that runs under win 32 and is built based on object-oriented Pascal language. Our simulator runs on Pentium 4, 2.66 GHZ, $512 \mathrm{Mb}$. We have implemented both Quick sort and PSA on the same machine and we have performed different simulations. Our simulation results show that the performance of PSA is better than quick sort in term of running time (Table 1). 


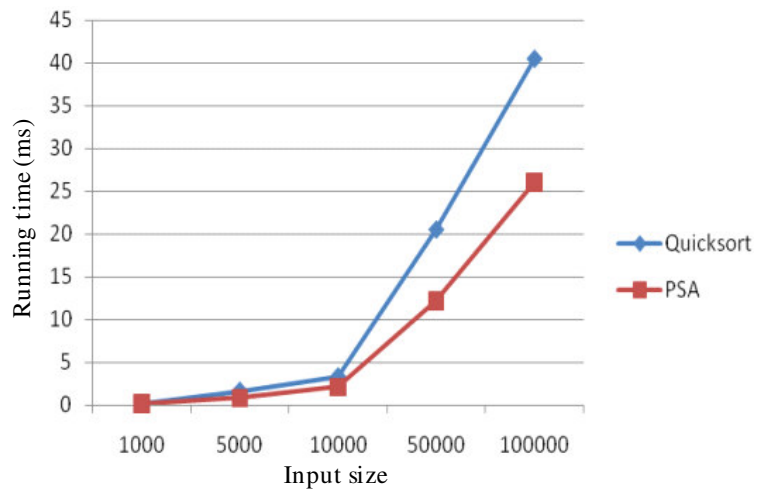

Fig. 5: The running time of both Quick sort and PSA algorithms

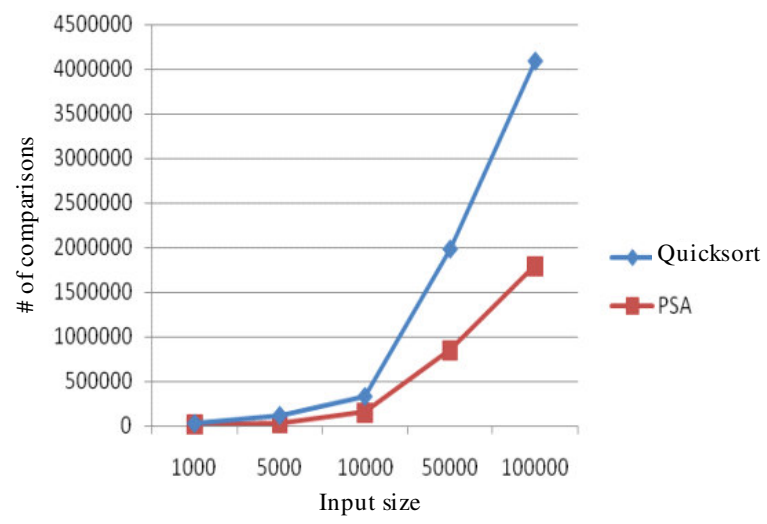

Fig. 6: The number of comparisons needed to sort the same input size in both Quick sort and PSA algorithms

For an input size 1000-5000 the performance of PSA is similar to that of Quick sort.

However, for a large data size (100.000) PSA outperforms Quick sort (Fig. 5). Figure 6, shows that there a big difference in the number of comparisons needed to sort the same input data between both Quick sort and PSA, where PSA requires a less number of comparisons with respect to Quick sort in all the input data sizes tested.

\section{DISCUSSION}

Our goal in this study is to propose an algorithm that performs better than Quick sort in term of running time. However the performance of quicksort is optimal only for a large input size. Analyzing the results presented in Table 1 we can see that the efficiency of our algorithm is about $73 \%$ for a small input size
Table 1: Comparison between the running time of Quick sort and PSA for different input size

\begin{tabular}{lcc}
\hline Input size & $\begin{array}{l}\text { Quick sort running } \\
\text { time }(\mathrm{ms})\end{array}$ & $\begin{array}{l}\text { PSA running } \\
\text { time }(\mathrm{ms})\end{array}$ \\
\hline 1000 & 0.234 & 0.171 \\
5000 & 1.640 & 0.829 \\
10000 & 3.313 & 2.139 \\
50000 & 20.485 & 12.203 \\
100000 & 40.460 & 26.090 \\
\hline
\end{tabular}

(1000 items), however the efficiency of our algorithm respectively to Quick sort is about $64.56 \%$ for a large input size and does not change if the input size increased from $\mathrm{n}$ to $10 \mathrm{n}$ (10000-100000 items). And that is accorded with our goal is to propose an algorithm simple that works in parallel and has a good efficiency for all input size.

\section{CONCLUSION}

In this study we have been proposed a sorting algorithm that uses less number of comparisons with respect to original Quick sort that in turn requires less running time to sort the same input data. Our algorithm is based on dividing the initial array in four partitions in a manner that during the partitioning process the subarrays are organized from the smaller to the bigger elements reducing in that the number of comparisons for the final merging operation. In addition it is used the heavily procedure that organize the nodes of each partition in a data structure useful for the searching operation. Our results show that PSA algorithm performs better than original Quick sort for the input data size tested.

Sorting algorithms can be classified into three categories: the first category is based on the number of comparisons in the worst and average and best case. The second category is based on the memory usage and the last category is based on the difference in behavior in the worst case and the average case.

$\mathrm{In}^{[2]}$, a comparison between three sorting algorithms (Heap sort ${ }^{[4]}$, Quick sort ${ }^{[3]}$ and Merge sort ${ }^{[5]}$ ) is done and a new algorithm is proposed that is a variant of heap sort (modified heap sort). The new algorithm requires nlogn- 0.788928 comparisons in the average case. The algorithm uses only one comparison at each node. With one comparison, the algorithm can decide which child of a node contains a larger element and this child is promoted to its parent position.

The four previous algorithms (heap sort, mergesort, quicksort, modified heap sort) were implemented to study their performance in term of execution time. For an input size 1000 the performance of heap sort is better 
than the other algorithms. However, for an input size from 5000-50000 quicksort outperforms the other algorithms in terms of execution time. However, for input size 100000 the modified heap sort requires a less running time to sort this input data. $\mathrm{In}^{[6]}$, a heap traversal algorithm is proposed that visits the node of a heap and stores its value in an auxiliary data structure with size $\lfloor n / 2\rfloor$, in a manner that the time for locating the next node for traversal is $\mathrm{O}(\log \mathrm{n})$ in the worst case. The main advantage of the algorithm is that the data structure used is small, which consequently helps in the searching operation. The algorithm takes a minheap as input and visits the heap in ascending order of the value stored in the nodes without making any changes to the structure or contents of the heap. The nodes that are being traversed are copied into another list, producing an ordered list of the values stored in the original heap. The algorithm is similar to an in-order traversal of a binary search tree. A comparison between the running time of generic Quicksort, optimized quicksort (optquicksort), heap sort, heap traversal of a binary tree (heapbt) and the proposed algorithm heap traversal using binary heap (heapbh) is done. The comparison results show that for an input size of 30000 the heapbh performs slightly better than heap sort. An another sorting algorithm that reduces the cache size is proposed $\mathrm{in}^{[7]}$, the proposed dualheap sort algorithm partitions recursively the subheaps in half until the subheaps become small to partition any more, then the array is sorted. The algorithm has different advantages in term of more operations and cache size. In the best case where the input is already sorted the dualheap sort performs no move operations and nlogn comparisons. In addition, the continued partitioning of the subheaps decreases the cache size needed in each step. The algorithm constructs two opposing subheap and then exchanges values between them until the larger values are in one subheap and the smaller values are in the other. The results presented in ${ }^{[7]}$ show that dualheap sort performs about (50\%) more comparisons and move operations than heap sort but the memory size needed is smaller. $\mathrm{In}^{[8]}$, a variant of heap sort is proposed that uses a new data structure for pairs of nodes of which can be simultaneously stored and processed in a single register. The algorithm sorts pair of elements in ascending order. By $\mathrm{n} / 2$ comparisons, all larger elements of each pair $2 \mathrm{i}, 2 \mathrm{i}+1$ can be brought to even positions. To construct the new data structure of size $\mathrm{n} / 2$ elements it needs $(1+\alpha / 2) \mathrm{n}$ comparisons where $\alpha>0$, in contrast to weak heaps ${ }^{[9]}$, that takes $n-1$ comparisons. In this data structure, during swapping it must swap nodes containing pair of elements with similar nodes at no extra cost. After constructing the heap, in the sorting phase the active elements (the even elements are called active and the other ones dormant) in the root will be placed in the last unsorted position and perform heavily or heap adjustment. This is to find out the larger or smaller key between the 2nd key of the 1st pair and the 1st key of the last pair. In the new data structure each key require three movements. The experimental results indicate that the new data structure for heaps results in better performance of heap sort algorithm in term of number of comparisons. $\mathrm{In}^{[10]}$, is proposed ultimate heap sort that is a variant of heap sort that sorts $n$ elements in $\theta\left(n \log _{2}(n+1)\right)$ time in the worst case by performing at most $\log _{2} n+\theta(n)$ key comparisons and $\log _{2} n+\theta(n)$ element moves. The algorithm transforms the heap on which it operates into two-layer heap which keeps small elements at the leaves. The two layer heap works as follow: first it finds $2 \mathrm{~d}$ the largest key of the elements in the array where denoted as $\mathrm{x} . \mathrm{x}$ is the largest power of 2 smaller than or equal to $\mathrm{n}$. Then, it partitions the array $\mathrm{A}[1 . . \mathrm{n}]$ into three pieces: $\mathrm{A}[1 \ldots \mathrm{r}], \mathrm{A}[\mathrm{r}+1 \ldots \ldots \mathrm{r}+\mathrm{e}]$ and $\mathrm{A}[\mathrm{r}+\mathrm{e}+1, \ldots \ldots \mathrm{n}]$, in a manner that the key of every element in the three partitions is larger than, equal to and smaller (respectively) to $\mathrm{x}$. Then the array $\mathrm{A}[1 \ldots . \mathrm{r}]$ is arranged into a heap using standard heap construction algorithm. In ultimate heap sort, the input array to be sorted of size $\mathrm{n}$ is sorted in $\mathrm{d}-1$ rounds. Where $\mathrm{d}=\mid \log _{2}$ $(\mathrm{n}+1) \mid$. In each round one half of the elements are sorted. In the first phase of the ith round, $I=\{1 \ldots$ d- 1$\}$ the remaining $n_{i}$ elements are built into a two layer heap. In the second phase of the ith round, the ri elements with a key larger than $\mathrm{x}$ are removed from the heap. The removed elements are exchanged with the last ri elements of the heap. In the third phase of the ith round, $\mathrm{n}_{\mathrm{i}}-2 \mathrm{di}$-ri of the elements with a key equal to that of $\mathrm{x}$ are gathered together and moved at the end of the sub-array containing the remaining elements. The computations carried out in each round are the following:

- Rearrange $\mathrm{A}[1 . . \mathrm{n}]$ into a two layer heap

- For $\mathrm{n}$ steps-1 $\rightarrow \mathrm{j}$ until $\mathrm{n}-\mathrm{l}$ do: exchange $\mathrm{A}[1]$ and $A[j]$, remake $A[1 \ldots \ldots . . . j-1]$ into heap

\section{REFERENCES}

1. Cormen, T., C.E. Leiserson, R.L. Rivest and C. Stein, 2001. Introduction to Algorithms. 2nd Edn., MIT Press and McGraw-Hill. ISBN 10: 0262-03293-7.

2. Sharma, V., S. Singh and K.S. Kahlon, 2008. Performance study of improved HeapSort algorithm and other sorting algorithms on different platforms. Int. J. Comput. Sci. Network Secur., 8: 101-105. http://paper.ijcsns.org/07_book/200804/20080415. pdf. 
3. Hoare, C.A.R., 1962. Quick sort. Comput. J., 5: 10-15. DOI: $10.1093 /$ comjnl/5.1.10.

4. Wegener, I., 1990. Bottom-up heap sort a new variant of heap sort beating on average quicksort. Proceeding of the Mathematical Foundations of Computer Science, Aug. 27-31, Springer-Verlag Inc., Banska' Bystrica, Czechoslovakia, New York USA., pp: 516-522. http://portal.acm.org/citation.cfm?id=90256.

5. Knuth, D.E., 1998. The Art of Programming Sorting and Searching. 2nd Edn., Addison-Wesley Professional, pp: 780. ISBN: 0-201-89685-0.

6. Mao, L.J. and S.D. Lang, 2003. An empirical study of heap traversal and its applications. Proceedings of the 7th World Multi Conference on Systematic, Cybernatics and Informatics, July 27-30, OrlandoFlorida, USA., $\quad$ 167-171. http://www.cs.ucf.edu/csdept/faculty/lang/pub.html.

7. Sepesi, G., 2007. DualHeap sort algorithm: An inherently parallel generalization of heapsort. http://adsabs.harvard.edu/abs/2007arXiv0706.2893S
8. Shahjala and M. Kaykobad, 2007. A new data structure or heap sort with improved numbers of comparisons. Proceedings of the 1st Workshop on Algorithms and Computation, Feb. 12-12, Dhaka, Bangladesh, pp: 88-96. http://teacher.buet.ac.bd/saidurrahman/walcom200 7/proceedings/shahjalal.pdf.

9. Duton, R.D., 1993. The weak heapsort. BIT Numer. Math. J. Part I Comput. Sci., 33: 372-381. http://www.springerlink.com/content/x442k4973j5 $242 \mathrm{p} 0 /$.

10. Katajainen, J., 1998. The ultimate heapsort. Proceedings of the Computing: The 4th Australian Theory Symposium Australian Computer Science Communications, Springer-Verlag. Singapore, pp: 87-95. http://citeseerx.ist.psu.edu/viewdoc/summary?doi=1 0.1.1.33.4727. 